\title{
THE BASIC HYPOTHESIS FOR MECHANOTRANSDUCTION IN SENSORY RECEPTORS
}

\author{
Ch. Chouchkov, Iv. Maslarski* \\ Department of Anatomy, Histology, Pathology and Forensic Medicine, Faculty of Medicine, \\ SU "St. Kliment Ohridski", Sofia, Bulgaria
}

\begin{abstract}
One of the most involving problem in the nerve tissue organization is the process of information transduction in the sensory receptors and the associated with them sensory cells. The aim of the present report is to review and discuss the data during the latter twenty years about the localization of molecules recently localized in different structural elements of sensory corpuscles and their possible role in the process of mechanotransduction. The most important obligatory parts in the process of mechanotransduction of all sensory receptors are their non-myelinated parts and their bulbous ends or so called nerve endings, like Pacinian and Meissner corpuscles, Krauses bulb, Golgi-Mazzoni corpuscle and Merkel disk.

In conclusion, it is still a matter of elucidation in the future to precisely localize the proteins making up the mechanosensitive ion channels. There also exist difficulties regarding the correlation of the physiological with morphological data due to the fact that the receptor axolemma is surrounded by complex cellular structures whose isolation is hard to perform.
\end{abstract}

Key words: Merkel disk, Pacinian corpuscle, Schwann inner core complex, neurotransmission

\section{INTRODUCTION}

One of the most intriguing problem in the nerve tissue organization is the process of information transduction in the sensory receptors and the associated with them sensory cells. We are still unable to explain and perceive the molecular processes in the initiation of receptor potentials derived in the conduction of primary sensation such as touch, mechanical pressure and vibration. On the other hand, the role and significance of sensory perception is not only limited to the primary sensation but is also has great importance for a number of involuntary physiological events. The perception of sensation become possible only if a physical stimulus has been detected and transduced into a signal to the primary sensory neuron. The process by which a physical stimulus is converted into a ionic current and receptor potential is termed

\footnotetext{
*Correspondence to: Ivan Maslarski, Departmant of Anayomy, histology, pathology and forensic medicine - Faculty of medicine, SU "St. Kliment Ohridski”, 1407 Sofia, 1 Kozyak str., Bulgaria, Email: maslarsky@gmail.com, Phone: $+359882000809$
}

mechanotransduction. The sensory receptors, responsible for the process of mechanotransduction are designed as mechanoreceptors. All known mechanoreceptors responds to motion and deformation in a different way but the basic structural principals in their functional responces could be summarized in two postulates: firstly, the mechanosensitive transducers reside in the nonmyelinated endings of the receptor nerve fibers and secondly, the receptor selectivity depends as much to the receptor structures that surrounds such of these endings as to the trancducer inself.

The aim of the present report is to review and discuss the data during the latter twenty years about the localization of molecules recently localized in different structural elements of sensory corpuscles and their possible role in the process of mechanotransduction.

The somatovisceral sensory receptors can be grouped by their location in the skin, skeletal muscles, joints, or viscera. As general examples, I shull speak about mechanoreceptors in the skin which are encapsulated as Pacinian, Meissner, Krause corpuscles or unencapsulated or so colled free nerve endings. 
Before report the contemporary data concerning the hypotetic process of mechanotranduction I show you the general structure of both groups of cutaneous mechanoreceptors very quickly according to impregnational method. The most important obligatory parts in the process of mechanotransduction of all sensory receptors are their non-myelinated parts and their bulbous ends or so calld nerve endings Pacinian corpuscle, Meissner corpuscles, Krauses bulb, GolgiMazzoni corpuscle, uncapsulated free nerve endings, Merkel disk I show the non-myelinated nerve fibres and their endings (Figures 1-6). Now I show you the electron microscopical structure of Pacinian corpuscle, because Pacinian corpuscle possesses the basic structural elements of most other mechanoreceptors, so it serves as a reliable model for deriving physiological and biophysical data that are valid for the sensory mecanoreceptor group and the process of mechanotransduction.

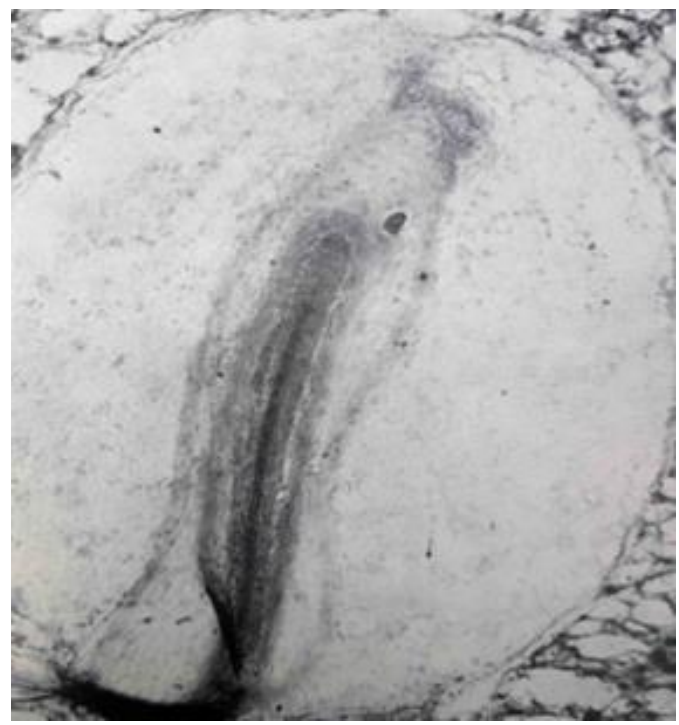

Figure 1. Pacinian corpuscle

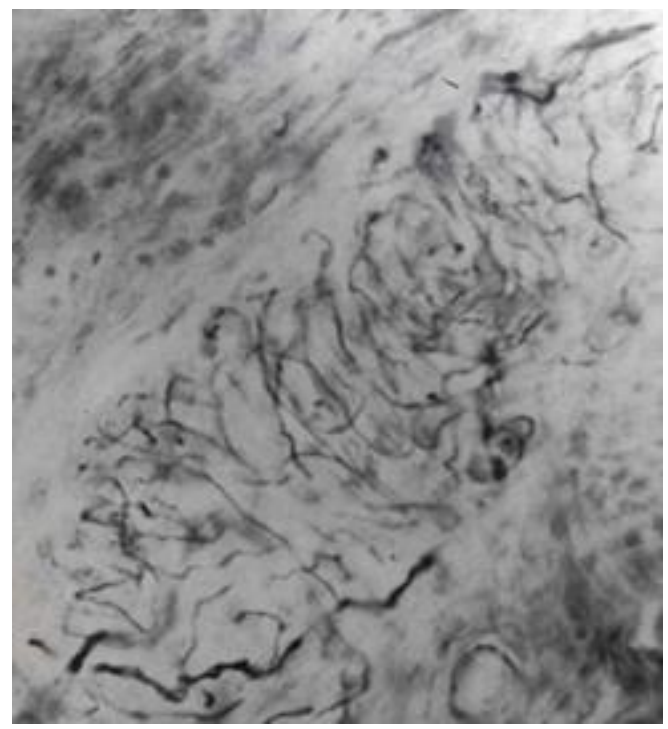

Figure 2. Meissner corpuscles

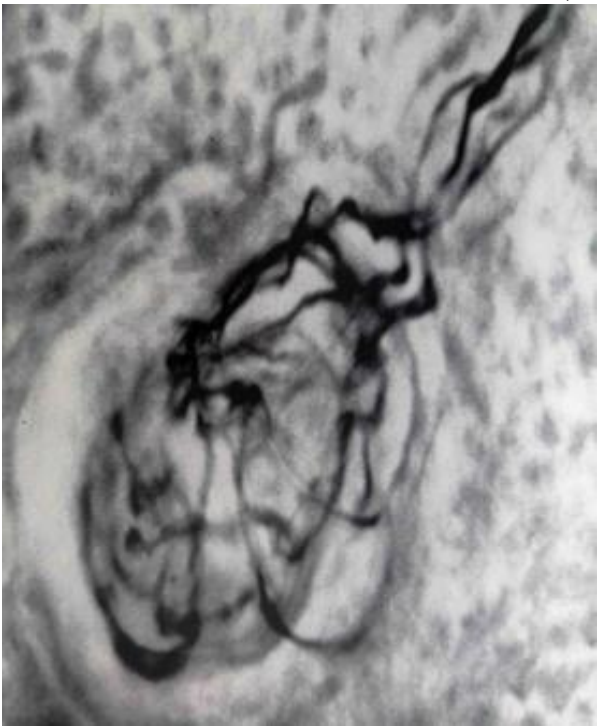

Figure 3. Krauses bulb

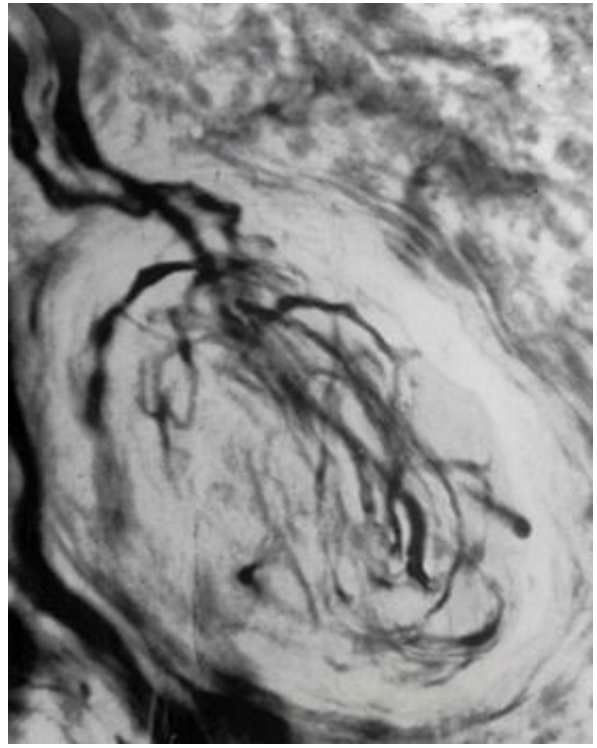

Figure 4. Golgi-Mazzoni corpuscle

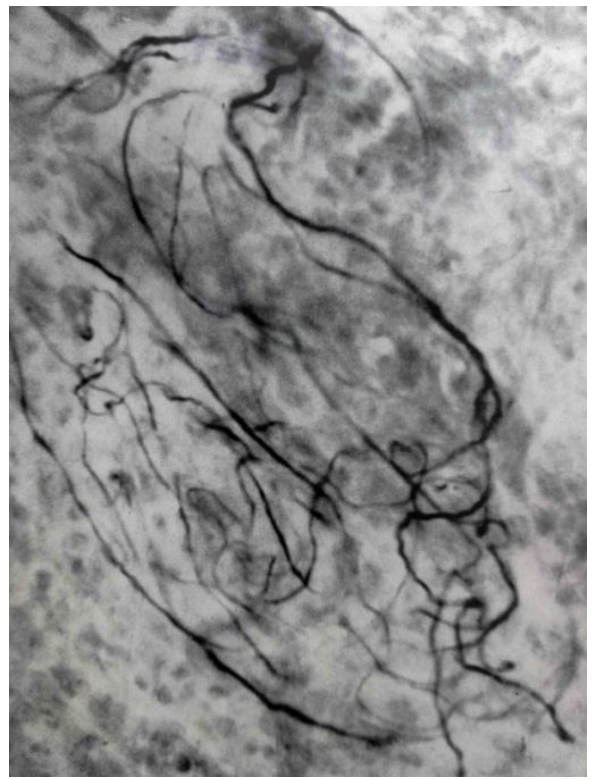

Figure 5. Uncapsulated free nerve endings 


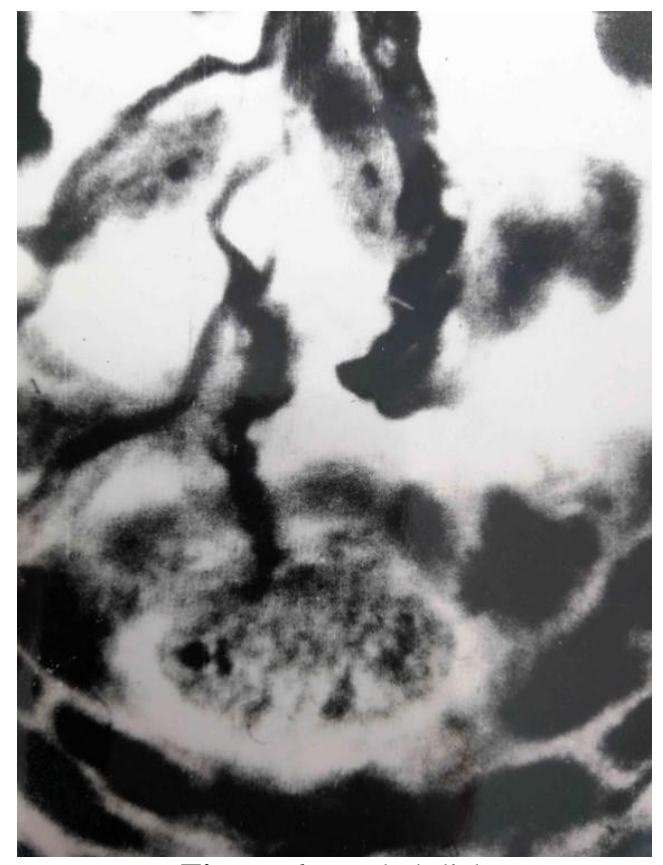

Figure 6. Merkel disk

Pacinian corpuscle consists of a capsule, a narrow subcapsular space, axially situated modified Scwann cells and the nerve fiber (Figure 7). The latter after entering the corpuscle could be divided on three parts: myelinated-between the capsule cells, nonmyelinated- between Schwann cytoplasmic lamellae and a large terminal nerve ending between lamellae and intercellular space

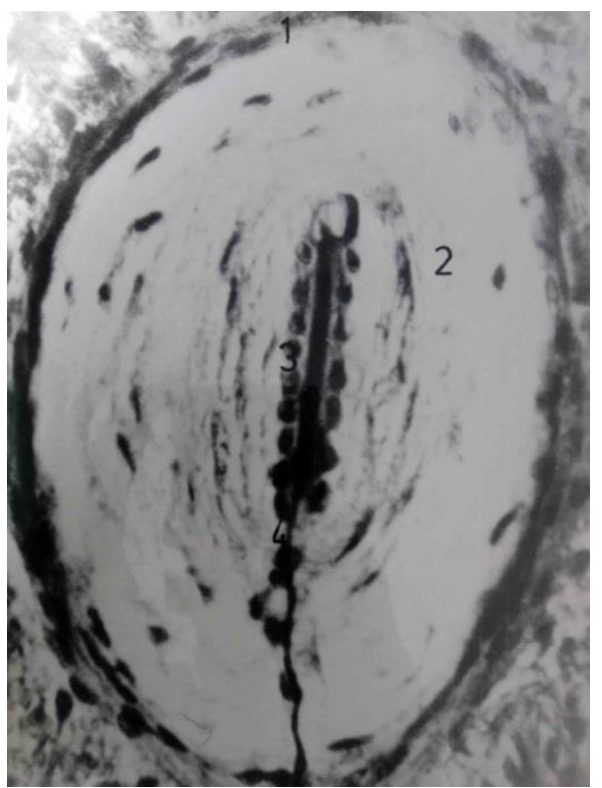

Figure 7. Pacinian corpuscle consists of a capsule

The fundamental cellular unit of the capsule is the perineural cell (Figure 8). In most capsulated mechanoreceptors the perineural cells are organized in layers where the cells are linked by tight junctions or desmosome-like contacts. The cytoplasm contain actin
CHOUCHOV CH., et al.

filaments, coated and pinocytotic vesicles usually densely packed on both sides of the plasmalemma (Figure 9). These vesicles are obligatory structure also for the inner core lamellae of the Schwann cells (7).

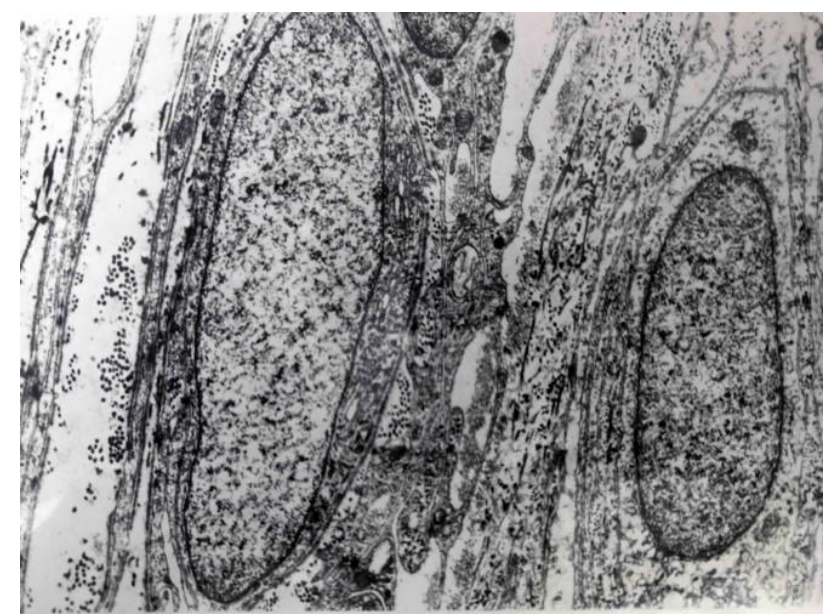

Figure 8. The fundamental cellular unit of the capsule

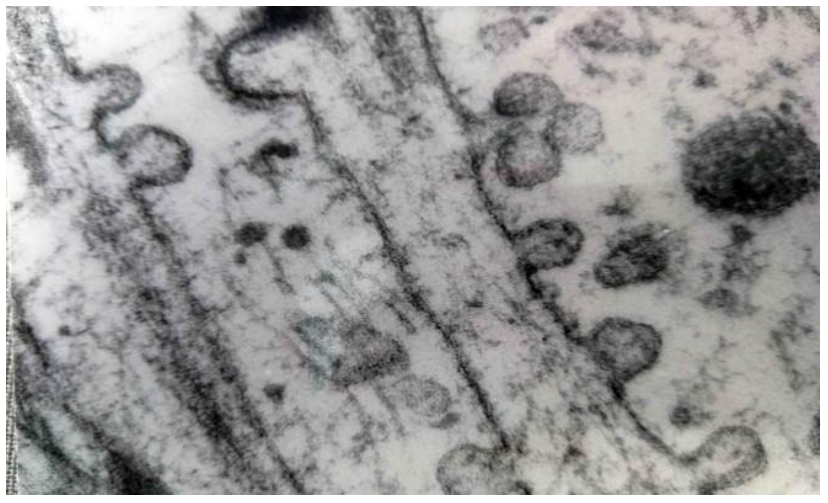

Figure 9. Coated and pinocytotic vesicles

What is the respective role of the capsule? The experiments prove that the layered lamellae function as an extremely selective cascade of high-pass filters. When a mechanical stimulus is transmitted through the capsule, the lamellae permit only a dynamic component of a mechanical pulse to reach the non-myelinated parts of the nerve fiber. The capsular layers that are sealed by tight junctions and desmosome contacts further act as a bloodnerve barrier that isolated the Schwann complex with the nerve fiber from the surrounding environment. If the capsule is removed the corpuscle changes from a rapidly -into a slowly adapting receptor. In the latter three years was proved immunohistochemically the presence of the neurotrophins brain derived factor and its receptor primarily into axon but also in the Schwann complex and even in the capsule cells. The authors suggest the existence of a 
retrograde trophic signaling mechanism to maintain functional integrity of sensory neurons supplying Pacinian corpuscle $(3,4,8)$.

The Schwann-inner core complex is built from lamellae of the Schwann modified cells project inward and give rise to crescent-like lamellae (Figure 10). They contain a sparce accumulation of microtubules, intermediate filaments, a few mitochondria and numerous coated and pinocytotic vesicles

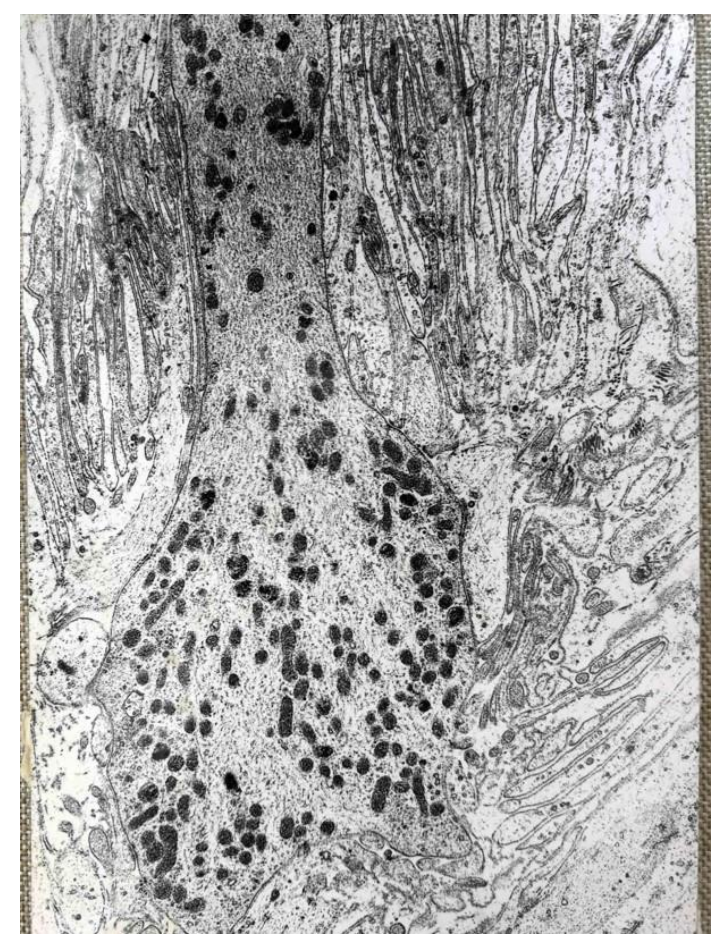

Figure 10. The Schwann-inner core complex

The Schwann inner core complex probably regulate the environment surounding the nonmyelinated neurite and its ending also prevent the movement of fluids along the lamellae and act as a selective and permeability barrier for ions and molecules. The lamellae modified the effect of the pressure pulse upon the neurite depending on the direction along which stimulus is applied.

The most intriguing feature of the Pacinian corpuscle which is responsible for the initiation and generation of the receptor potential is the non-myelinated part of the nerve fiber and its ending (Figure 10). The neuroplasma contains many mitochondria, neurofilaments, microtubules, different kind of vesicles, lysosomes, multivesicular bodies and glycogen granules. Special attention deserve the axoplasmic extensions as finger-like projections or fillopodia which I described during 20 years in all mechanoreceptors as the obligatory structures in the non-myelinated nerve fiber and nerve endings. These filopodia are only covered with a basement membrane. Sometimes they branch and give the nerve ending a mace-like appearance. Their length is moving between 0.5 and $2 \mathrm{~nm}$. Their neuroplasm contained interconnected filaments. Using the freeze-fracture technique we have established a denser accumulation of intramembranous particles in this region comparing with the resting parts of the nerve fiber. Immunohistochemically, the filopodia contain actin in a much greater density than that seen in the neurite proper (Figure 11). Lack of other cytoplasmic structures except for neurofilaments is highly reminiscent of the composition of stereocilia found on vestibular and auditory hair cells. Since stereocilia have been shown to play a role in hair-cell mechanotransduction it is possible that the filopodia in the non-myelinated nerve fiber and its endings are significantly involved in the process of mechanotransduction (4).

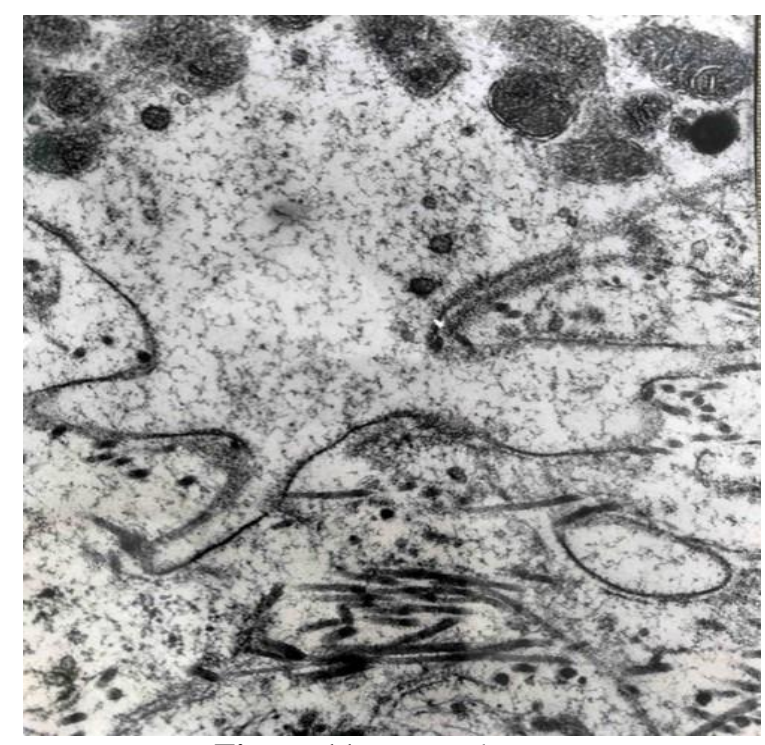

Figure 11. Neuroplasma

Independently of the many structural, physiological and biochemical investigations published in the literature in the last 50 years the main problem for the nature of the transduction process is open - is this process mechanosensitive or chemical. The most authors consider that the voltage-gated and non-voltede-ion channels initiate mechanotransduction. They suggest that mechanotransduction events are formed by stretch-activated channels selectively sensitive predominantly to sodium but also to potassium. Consistently, the alfa-subunit of rat type I and type II voltage gated Sodiumchannels are present in the central axon, the 
inner core and capsule lamellae. The authors suggest that both transduction and action potentials generation may be available to the axon via transport from the mechanism presumably parallel to axoplasmic transport. On the other hand, genetic, molecular, imaging and electrophysiological studies have demonstrated that channel complexes of the so-called epithelium sodium channel family of ion channel might be involved in mechanotransduction. Ion channel subunits of this family are not gated by voltage but by other stimuli, including mechanical modifications proposed as mechanotransducers $(5,6)$.

On the other hand, the possible classical neurotransmission can't be excluded for the genesis of action potential. Synaptic-like activity has been proposed in some publications together with the occurrence of glutamate, glutamate receptors and transporters in the Schwann core lamellae near the neurite. These findings argue for a chemical possibly bidirectional interaction between the lamellar cells and axon. In the last picture you see collection of different kind of vesicles in the axon near the cytoplasmic processes of the Schwann cells and the presence of well developed contact between them. But till now there is no histochemical evidence for the existence of a known neurotransmitter, the clustering and the presence of vesicles is no typical for synaptic vesicles and very important - the possibility for a release of a chemical transmitter to activate the mechanotransductive channels is disavoided because of the short latencies between the stimulus onset and the receptor potential initiation $(1,2)$.

\section{CONCLUSION}

It is still a matter of elucidation in the future to precisely localize the proteins making up the mechanosensitive ion channels. There also exist difficulties regarding the correlation of the physiological with morphological data due to the fact that the receptor axolemma is surrounded by complex cellular structures whose isolation is hard to perform. It is necessary to provide special experimental procedure for identifying the mechanosensitive ion channels and to characterisze the proteins making up their complex inner structure. It is our great hope that the future progress in the field of molecular biology will make it possible to unravel the enigma that dated back more than two senturies. Shortly speaking the title of present report is open for prove and discussion.

\section{REFERENCES}

1. Cabo, R., Alonso P., San José, I, Vázquez, G. Brain-derived neurotrofic factor and its receptor TrkB are present, but segregated, within mature cutaneous Pacinian corpuscles of Macaca fascicularis., Anat. Rec. (Hoboken).,298(3):624-9,2015.

2. Calavia, M., Montaño J., Suárez G., Differential localization of Acid-sensing ion channels 1 and 2 in human cutaneus pacinian corpuscles. Cell Mol Neurobiol. 30(6):841-8, 2010.

3. Chouchkov, C, Andreev, D, Dandov, A.. Localization and distribution of laminin in the basal lamina of certain mechanoreceptors - an immunogold study. Somatosens Mot Res 20:265-270, 2003.

4. Drummond, H., Abboud F., Welsh, M. Localization of beta and gamma subunits of $\mathrm{ENaC}$ in sensory nerve endings in the rat foot pad. Brain Res 884:1-12, 2000.

5. Marquez, J., Perez, M., Naves, F., Vega J. Effect of spinal cord and peripheral nerve injury on human cutaneous sensory corpuscles. An immunohistochemical study. $J$ Peripher Nerv Sys 2:49-59, 1997.

6. Mesa, G., Piqueras, G., et al., Merkel cells and Meissner's corpuscles in human digital skin display Piezo2 immunoreactivity. $J$ Anat. 231(6):978-989, 2017.

7. Nolano, M., Provitera, V., Crisci C., Stancanelli, A., Wendelschafer-Crabb G, Kennedy, WR, Santoro, L. Quantification of myelinated endings and mechanoreceptors in human digital skin. Ann Neurol 54:197-205, 2003.

8. Vega, J., Suarez, O., Montano, J., et al., The Meissner and Pacinian sensory corpuscles revisited new data from the last decade. Microscopical research technique, 72(4): 299-309, 2009. 
CHOUCHOV CH., et al. 